\title{
Bariatric surgery improves knee function and not knee pain in the early postoperative period
}

\author{
Amre Hamdi ${ }^{*} \mathbb{D}$, Alia T. Albaghdadi ${ }^{1}$, Bayan Ghalimah ${ }^{1}$, Abdullah Alnowiser $^{2}$, Anas Ahmad² and Abdulmalik Altaf ${ }^{3}$
}

\begin{abstract}
Background: Obesity remains the strongest predictor of knee osteoarthritis (OA). Studies have reported improvement in knee pain and function post-bariatric surgery secondary to weight loss and reduced mechanical loading, yet others found increased rates of total knee arthroplasty (TKA) in that patient population. To address this controversy, our study aimed to further assess the effect of surgically induced, "rapid" weight loss on knee pain and function.

Methods: Obese patients with chronic knee pain, who were undergoing bariatric surgery, were enrolled and surveyed preoperatively and 3 months postoperatively. Our outcome measures were knee pain and knee function, assessed by a knee injury and osteoarthritis outcome score (KOOS). The paired $t$ test was used to compare pre- and postoperative KOOS scores. Pearson correlation coefficient was used to test the correlation between change in body mass index (BMI) with knee function, pain, and stiffness.

Results: A total of 30 patients was included in the study. The mean age was 35 years, with a mean preoperative BMl of 42.8. The mean difference in BMl at 3 months was 8.4 (SD3). There was a significant improvement in KOOS, $-23.2( \pm$ 20) points, $p<0.01$, most pronounced in knee function related to sport activities, with a difference of -22.6 points, $p<$ 0.01 . Knee pain scores improved but did not reach statistical significance.

Conclusion: Surgically induced rapid weight loss significantly improved knee function, particularly related to sports. However, there was no change in knee pain. This may be related to increased high-impact knee exercises and reduced lean mass. Tailored exercise programs for bariatric surgery patients postoperatively, may improve symptoms and decrease the need for knee replacements in the long term.
\end{abstract}

Keywords: Knee pain, Arthritis, Knee function, Bariatric surgery, Rapid weight loss

\section{Background}

Knee osteoarthritis (OA) is one of the leading causes of knee pain and disability. A substantial escalation in the prevalence of knee pain and symptomatic knee OA was witnessed in the past two decades. The rates were found to double in women and triple in men [1]. The overall prevalence of knee pain in the US adult population is $20 \%$, with over 61 million people affected [2], and a projected increase in prevalence to $25 \%$ by the year 2030 [3]. National data on the prevalence of knee pain and knee OA in Saudi Arabia is

\footnotetext{
* Correspondence: amrehamdi@yahoo.com

${ }^{1}$ Department of Orthopedic Surgery, Faculty of Medicine, King Abdulaziz

University, P.O Box 80215, 21589 Jeddah, Kingdom of Saudi Arabia

Full list of author information is available at the end of the article
}

limited; however, significant numbers were reported ranging from 13 to $60 \%$ of studied sample populations in some regions of the country $[4,5]$, despite being a country with a relatively young population. Older age, female gender, smoking, diabetes, knee injury, and physical workload [6] such as squatting, kneeling, and lifting are among many known risk factors that contribute to the development and progression of knee OA. Nevertheless, obesity (BMI $>30 \mathrm{~kg} /$ $\mathrm{m}^{2}$ ) remains the strongest predictor of OA [7], while it is a modifiable risk. The mechanism by which obesity leads to knee joint damage and hence symptomatic OA is a complex process that includes biomechanical factors and an inflammatory process. Increased joint loading on mechanoreceptors 
overlying articular cartilage trigger a cascade of events resulting in cartilage degeneration. In addition, metabolic derangements in obese individuals lead to a systemic inflammatory response and hence disordered cartilage homeostasis [8]. Loss of hyaline cartilage is often accompanied with bony remodeling and periarticular muscle weakness. This results in synovitis, periosteal irritation, and therefore knee pain [9].

With lifestyle modification, every $1 \mathrm{~kg}$ in weight reduction was found to be associated with more than twice the reduction in peak knee load [10]. Further, a 2 -unit reduction in BMI over a 10-year period was associated with decreased odds for developing symptomatic knee OA by over 50\% [11]. Amidst the alarming rates of the obesity epidemic that affects over $28 \%$ of the Saudi population [12], more people are resorting to a rather more "rapid" method of weight loss-bariatric surgery. Bariatric surgery proved to be a success, not only in weight reduction, but also in managing co-morbid conditions associated with obesity, such as diabetes, hypertension, and obstructive sleep apnea [13]. As one might expect, significant improvement in knee pain and function following bariatric surgery was also reported in some studies despite using different measurement instruments [1416]. This was attributed to decreased mechanical loading and reduced markers of inflammation. Contrary to these findings, Trofa et al. [17] reported that bariatric surgery patients who are known to have arthritis are at an increased risk of requiring a total knee arthroplasty (TKA) following rapid weight loss. They suggested an active lifestyle on an already damaged knee as a possible culprit. In addition, the literature advocates for bariatric surgery as a staging tool prior to other non-bariatric surgical procedures in obese individuals [18], thereby lowering the risk and improving outcomes of a second indispensable surgery such as total knee replacement (TKR) [19].

To address the current controversy, we aimed at assessing the impact of rapid weight loss on the progression of knee pain and the development of OA in patients who undergo a bariatric procedure. We hypothesize that patients with a surgically induced rapid weight loss engage in more knee-loading activities, resulting in an "apparent" improvement in knee function but not knee pain. Further, we chose to select patients who suffer from knee pain, but without an identifiable cause or any radiographic evidence of OA, that is, who are not candidates for TKR, to limit the risk of bias in temporality.

The objectives of this study were to:

a. Assess the change in knee pain and function preand postoperatively in patients undergoing bariatric surgery using the knee injury and osteoarthritis outcome score (KOOS) [20, 21].

b. Determine the clinical significance of the change in the KOOS score in our patient population relative to a predetermined minimal clinically important change (MCIC) [22] of 8-10 points.

\section{Methods}

We obtained ethical approval from the Institutional Review Board at King Abdulaziz University Hospital (KAUH), Jeddah, Saudi Arabia (Reference No. 322-14). We then took an informed consent from all patients prior to their enrollment into the study. We carried out the study in a single tertiary care center over a period of 6 months (July-December 2015). Patients were enrolled from the bariatric surgery clinic.

We used a quasi-experimental study design, where we surveyed our enrolled subjects preoperatively and 3 months postoperatively during their follow-up visit. Eligibility for enrollment were as follows: age > 18 years, candidate for a bariatric procedure as defined by the American Society for Metabolic and Bariatric Surgery (ASMBS) guideline [23] (BMI $\geq 40$ or $\mathrm{BMI} \geq 35$ with at least two obesity-related comorbidities), and the presence of self-reported chronic knee pain, without an identifiable pathology on clinical and radiographic evaluation. Patients who had a history of knee surgery and knee trauma and any evidence of $\mathrm{OA}$ and those on chronic use of nonsteroidal anti-inflammatory drugs (NSAIDS), or received intra-articular steroid injections, were excluded from the study.

Eligible patients underwent the usual pre-operative workup followed by either restrictive (sleeve gastrectomy) or a malabsorptive procedure (Roux-en-Y gastric bypass) depending on their initial weight, risks, and preference. A board-certified, experienced bariatric surgeon operated on all enrolled patients. Standardized pre- and postoperative protocol was used for all patients. No adverse events were noted. The mean length of hospital stay was 3 days. There were no intra-operative or postoperative complications.

Our primary outcomes were knee pain and knee function. These were assessed by the KOOS [20, 21], which is a validated, self-administered survey that takes about $15 \mathrm{~min}$ to complete. It is composed of five subscales: Knee Pain, Stiffness, Function in activity of daily living (ADL) [24], Function in sport and recreation (Sport/Rec), and knee-related Quality of life (QOL). Each question is made up of a 5-point Likert-type scale. An Arabic-validated translation of the survey was given to patients [25]. All patients were fluent Arabic speakers. The KOOS subscale "sport and recreation function" is more sensitive and discriminative than the 
Table 1 Patient demographic characteristics

\begin{tabular}{ll}
\hline Patients demographics & N, \% (total 30) \\
\hline Age (mean, SD) & $35.6(9.3)$ \\
Gender & \\
$\quad$ Males & $6(20.0)$ \\
$\quad$ Females & $24(80.0)$ \\
Pre-op BMI (mean, SD) & $42.8(5.8)$ \\
Diabetes & $4(13.3)$ \\
History of smoking & $4(13.3)$ \\
Type of bariatric surgery & \\
$\quad$ Sleeve gastrectomy & $28(93.3)$ \\
RYG bypass & $2(6.7)$ \\
\hline
\end{tabular}

$B M I$ body mass index, Pre-op preoperative, RYG roux-en-Y

Western Ontario and McMaster University Osteoarthritis Index (WOMAC) subscales, and therefore, we decided to use the KOOS instrument [20, 21]. Data from surveys were then administered into a publicly available online software [26] to calculate the total KOOS and the subscale scores.

Statistical analysis was conducted using STATA [27], version 13.1. Categorical variables were expressed as a number and percentage. Data variables were then tested for normality. Continuous variables were expressed as mean (SD) for normally distributed variables and as median (IQR) for skewed distributions. Paired $t$ test was used to compare within group difference in pre- and postoperative mean scores. Pearson correlation co-efficient, $r$, was used to test correlations between independent variables. A twotailed $p$ value of $<0.05$ was considered statistically significant.

\section{Results}

\section{Patient demographics}

A total of 30 patients met our selection criteria and were enrolled in our study, of which 24 were females (80\%). Patient demographic characteristics are presented in Table 1. The mean age of patients was 35 years (SD 9), with a mean BMI of 42.7 (SD 5.73). Four patients were diabetic (13\%), and 4 had a history of tobacco use (13\%); 2 were current smokers, and 2 were ex-smokers. Patients had no history of trauma or knee surgery prior to the bariatric surgery. The majority of patients underwent sleeve gastrectomy ( $N 28,9 \%)$, and 2 patients underwent gastric bypass surgery. No other bariatric procedures were done.

\section{Knee function and knee pain}

As shown in Table 2, there is a significant reduction in BMI 3 months postoperatively, with a mean difference of 8.4 (SD 3) units, $p<0.01$, at $95 \%$ CI. Further, there is an overall significant reduction in the mean KOOS score, with a mean difference of $-23.2( \pm 20)$ points, $p<0.01$. The drop was most pronounced in knee function related to sport activities, with a difference of -22.6 points, $p<0$. 01 . Knee pain ( $p 0.147)$, stiffness ( $p 0.694)$, and daily function ( $p$ 0.167) improved, but not significantly from the preoperative period (Fig. 1). There were no difference in scores with regard to age, gender, diabetes, smoking status, or the type of bariatric surgery performed.

Clinical significance of the improvement in the KOOS score was determined using a pre-defined value of the minimal clinically detectable change (MCIC of 10) [22] to dichotomize the change in KOOS score. Sixty-three percent of patients ( $n$ 19) had a clinically significant improvement in their KOOS scores.

\section{Correlation between change in BMI with knee function, pain, and stiffness}

As shown in Table 3, the change in BMI was positively correlated with all KOOS parameters. Despite the lack of statistical significance, the highest correlation was seen in the improvement in knee stiffness and sport function with the change in weight. Contrary, the least parameter correlated with the change in weight was the change in knee pain ( $r$ 0.0039, $p$ 0.983) (Fig. 2).

\section{Discussion}

Knee pain is an increasingly common complaint, particularly in obese individuals. Weight reduction lessens

Table 2 Paired $t$ test on BMI and KOOS pre- and post-op

\begin{tabular}{llll}
\hline & Pre-op (mean, SD) & Post-op (mean, SD) & $p$ value (95\% Cl) \\
\hline BMI & $42.8(5.7)$ & $34.4(5.3)$ & $<0.01$ \\
KOOS (total score) & $66.0(19.9)$ & $89.3(8.91)$ & $<0.01$ \\
Knee stiffness & $50.2(18.4)$ & $52.0(19.1)$ & 0.694 \\
Knee pain & $70.3(24.38)$ & $79.9(25.9)$ & 0.147 \\
Daily function & $67.2(26.51)$ & $78.5(28.9)$ & 0.167 \\
Sports function & $54.3(23.41)$ & $77.0(20.2)$ & $<0.01$ \\
QOL related to knee & $60.2(27.68)$ & $80.3(21.9)$ & 0.004 \\
\hline
\end{tabular}

Pre-op preoperative, Post-op postoperative, BMI body mass index, KOOS knee osteoarthritis outcomes score, QOL quality of life 


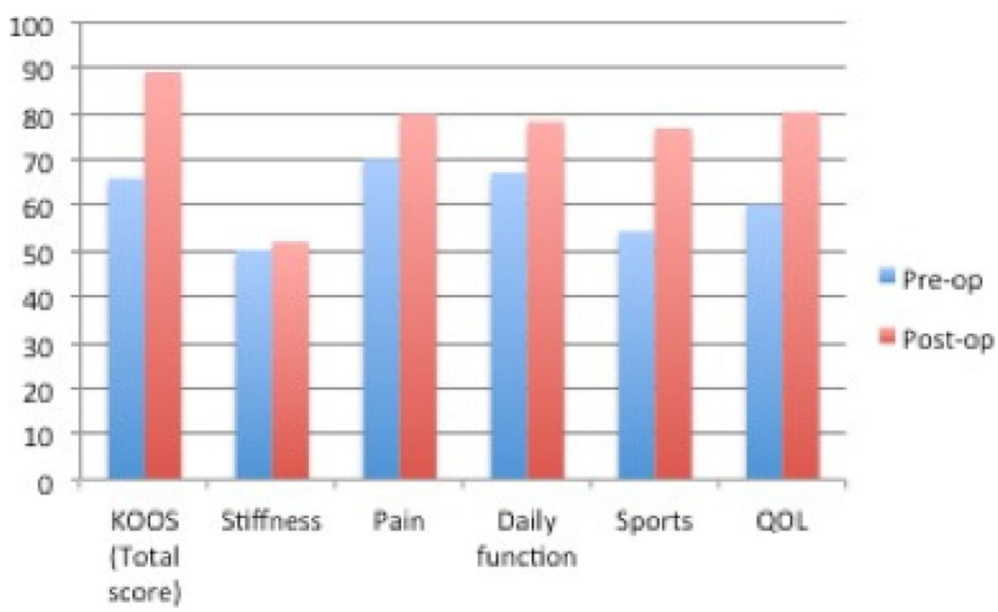

Fig. 1 Comparison between mean KOOS sub-scores pre- and post-bariatric surgery

mechanical loading on knees. Bariatric surgery, an effective approach to obesity that results in rapid weight loss, is further expected to positively affect knee symptoms. Multiple studies reported a substantial improvement in knee pain and function using different measurement instruments following bariatric surgery [14-16]. Albeit prior findings, Trofa et al. [17] found an increased risk of TKR in arthritis patients 25-48 months following bariatric surgery. To address the current controversy, in this study, we aimed at further assessing the impact of rapid weight loss on knee pain and function.

Our results demonstrated a significant improvement in knee function related to sports, but insignificant change in knee pain, which is contrary to previous findings in the literature. Our results are supportive of the finding of Trofa et al. [17] in that our patients may later become candidates for TKR. The patients' current more active lifestyle and increased demand on their knees since they are now more flexible due to weight loss as evident in our data may explain the insignificant change in their knee pain. The KOOS sport-related function subscale consists of five questions that assess the level

Table 3 Correlation between the change in BMI with the change in KOOS sub-scores

\begin{tabular}{llc}
\hline Change in KOOS score & $\begin{array}{l}\text { Pearson correlation }(r) \\
\text { with change in BMI }\end{array}$ & $p$ value \\
\hline KOOS (total score) & 0.0314 & 0.869 \\
Stiffness & 0.1894 & 0.316 \\
Pain & 0.0039 & 0.9836 \\
Daily function & 0.0842 & 0.6581 \\
Sports & 0.1196 & 0.529 \\
QOL & 0.0289 & 0.8794 \\
\hline $\begin{array}{l}\text { BMI body mass index, KOOS knee osteoarthritis outcomes score, QOL quality } \\
\text { of life }\end{array}$ &
\end{tabular}

of difficulty in squatting, running, jumping, twisting, and kneeling. All of which activities are known to cause patellofemoral pain syndrome (aka anterior knee pain) [28], which is a condition mainly described in active, young females [29]. Hence, the "rapid" transition from an inactive, sedentary life to a more active lifestyle may negatively impact long-term knee outcomes. Another possible explanation is that only a few patients maintain their lean body mass following bariatric surgery despite being active in exercise programs [30]. Moreover, rapid weight loss decreases dynamic and static muscle strength [31], and loss of quadriceps strength results in increased knee pain and the risk of arthritis development [31].

Given the importance of maintaining muscle mass and quadriceps strength with exercise, yet avoid activities that could induce even further damage with increased knee loading, a multidisciplinary care should be provided to patients postoperatively. There is a need to develop a standardized exercise protocol post-bariatric surgery to prevent further progression of knee pain and osteoarthritis and possibly avoid the need for a joint replacement.

Our study had several limitations; the lack of randomization limits causality; nevertheless, we attempted to control for confounders of knee pain by using a strict selection criteria. Patients were thoroughly evaluated prior to enrollment to exclude any identifiable knee pathology. In addition, the lack of significant improvement in knee pain may be secondary to our small sample size and short follow-up period. Although our follow-up period was limited to 3 months, reporting of data early in the postoperative period will allow us to examine sequential trends in the progression of our patients' knee outcomes relative to bariatric surgery. Further, in this study, we used the KOOS that has a sport-related knee function subscale more sensitive and discriminative than the WOMAC subscales. 


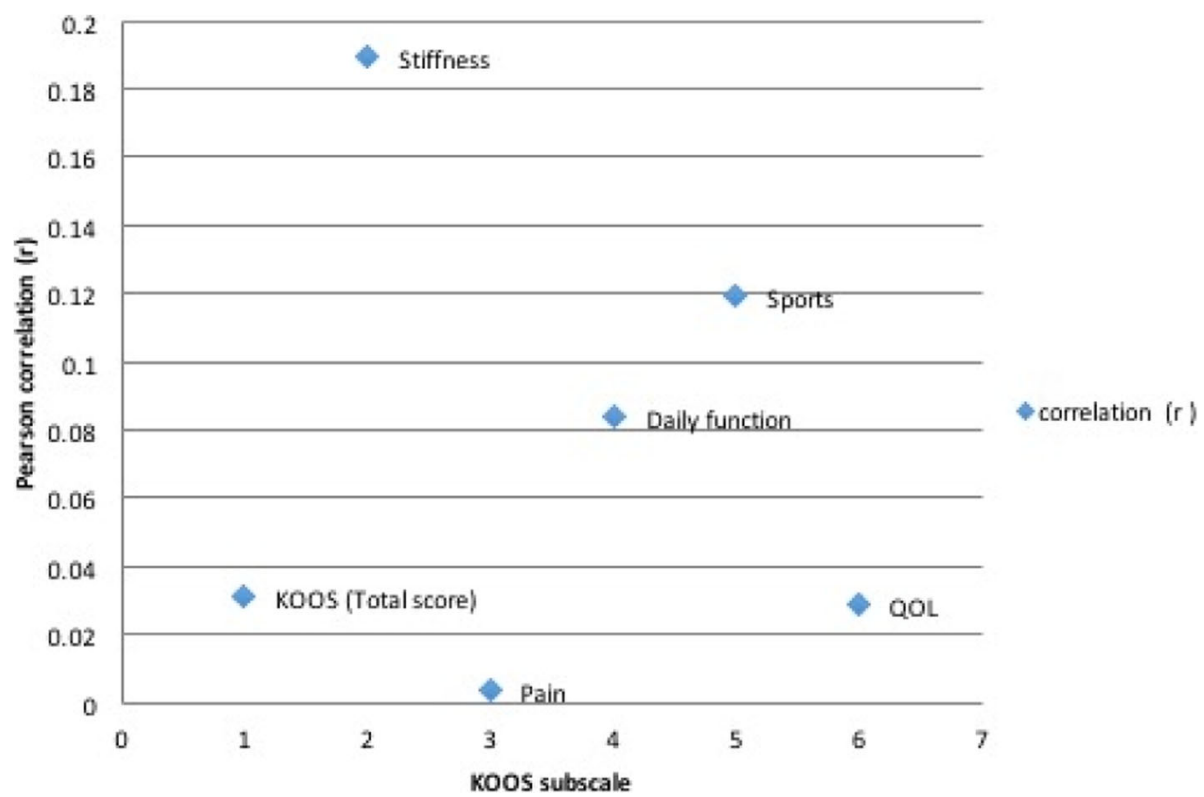

Fig. 2 Pearson correlation co-efficient ( $r$ ) of KOOS sub-scores with change in BMI

\section{Conclusion}

In conclusion, the rapid transition to an active lifestyle seen with massive weight loss post-bariatric surgery may halt the expected improvement in knee pain. There is a need for tailored exercise programs for bariatric surgery patients to strengthen their muscles, preserve their lean mass, and yet prevent progression of knee pain from excess strain.

\section{Abbreviations}

ADL: Activity of daily living; ASMBS: American Society for Metabolic and Bariatric Surgery; BMI: Body mass index; KAUH: King Abdulaziz University Hospital; KOOS: Knee Injury and Osteoarthritis Outcome Score; MCIC: Minimal clinically important change; NSAIDS: Nonsteroidal anti-inflammatory drugs; OA: Osteoarthritis; QOL: Quality of life; TKA: Total knee arthroplasty; TKR: Total knee replacement; WOMAC: Western Ontario and McMaster University Osteoarthritis Index

\section{Authors' contributions}

According to the definition given by the International Committee of Medical. Journal Editors (ICMJE), the authors listed above qualify for authorship based on making one or more of the substantial contributions to the intellectual content of (1) study conception and design [AH and AAltaf] and/or (2) analysis and interpretation of data [AAlbaghdadi, AAlnowiser, and AAhmad] and/or (3) participation in the drafting of the manuscript [AAlbaghdadi, BG, AH and AAltaf] and/or (4) critical revision of the manuscript for important intellectual content [AAlbaghdadi and BG]. All authors read and approved the final manuscript.

\section{Ethics approval and consent to participate}

The ethical approval is obtained from the Institutional Review Board at King Abdulaziz University Hospital (KAUH), Jeddah, Saudi Arabia with Reference No. 322-14. We then took an informed consent from all patients prior to their enrollment into the study.

\section{Competing interests}

The authors declare that they have no competing interests.

\section{Publisher's Note}

Springer Nature remains neutral with regard to jurisdictional claims in published maps and institutional affiliations.

\section{Author details}

'Department of Orthopedic Surgery, Faculty of Medicine, King Abdulaziz University, P.O Box 80215, 21589 Jeddah, Kingdom of Saudi Arabia. ${ }^{2}$ Faculty of Medicine, King Abdulaziz University, Jeddah, Kingdom of Saudi Arabia. ${ }^{3}$ Department of General Surgery, Faculty of Medicine, King Abdulaziz University, Jeddah, Kingdom of Saudi Arabia.

Received: 18 December 2017 Accepted: 3 April 2018

Published online: 11 April 2018

\section{References}

1. Nguyen US, Zhang Y, Zhu Y, Niu J, Zhang B, Felson DT. Increasing prevalence of knee pain and symptomatic knee osteoarthritis: survey and cohort data. Ann Intern Med. 2011;155(11):725-32. https://doi.org/10.7326/ 0003-4819-155-11-201112060-00004.

2. Simon LS. Relieving pain in America: a blueprint for transforming prevention, care, education, and research. J Pain Palliat Care Pharmacother. 2012;26(2):197-8. https://doi.org/10.1007/s13142-012-0110-2.

3. Hootman JM, Helmick CG. Projections of US prevalence of arthritis and associated activity limitations. Arthritis Rheumatol. 2006;54(1):226-9.

4. Al-Arfaj A, Al-Boukai AA. Prevalence of radiographic knee osteoarthritis in Saudi Arabia. Clin Rheumatol. 2002;21(2):142-5.

5. Al-Arfaj AS, Alballa SR, Al-Saleh SS, Al-Dalaan AM, Bahabry SA, Mousa MA, AlSekeit MA. Knee osteoarthritis in Al-Qaseem, Saudi Arabia. Saudi Med J. 2003:24(3):291-3.

6. Vrezas I, Elsner G, Bolm-Audorff U, Abolmaali N, Seidler A. Case-control study of knee osteoarthritis and lifestyle factors considering their interaction with physical workload. Int Arch Occup Environ Health. 2010;83(3):291-300. https://doi.org/10.1007/s00420-009-0486-6.

7. Silverwood V, Blagojevic-Bucknall M, Jinks C, Jordan JL, Protheroe J, Jordan KP. Current evidence on risk factors for knee osteoarthritis in older adults: a systematic review and meta-analysis. Osteoarthr Cartil. 2015;23(4):507-15. https://doi.org/10.1016/j.joca.2014.11.019.

8. Pottie P, Presle N, Terlain B, Netter P, Mainard D, Berenbaum F. Obesity and osteoarthritis: more complex than predicted! Ann Rheum Dis. 2006;65(11):1403-5.

9. Haviv B, Bronak S, Thein R. The complexity of pain around the knee in patients with osteoarthritis. Isr Med Assoc J: IMAJ. 2013;15(4):178-81.

10. Aaboe J, Bliddal H, Messier SP, Alkjaer T, Henriksen M. Effects of an intensive weight loss program on knee joint loading in obese adults with knee osteoarthritis. Osteoarthr Cartil. 2011;19(7):822-8. https://doi.org/10.1016/j.joca. 2011.03.006 
11. Felson DT, Zhang Y, Anthony JM, Naimark A, Anderson JJ. Weight loss reduces the risk for symptomatic knee osteoarthritis in women: The Framingham Study. Ann Intern Med. 1992;116(7):535-9.

12. Basulaiman M, El Bcheraoui C, Tuffaha M, Robinson M, Daoud F, Jaber S, Mikhitarian S, Wilson S, Memish ZA, Al Saeedi M, AlMazroa MA. Hypercholesterolemia and its associated risk factors_-Kingdom of Saudi Arabia, 2013. Ann Epidemiol. 2014;24(11): 801-8. https:/doi.org/10.1016/j.annepidem.2014.08.001.

13. Buchwald $H$, Avidor $Y$, Braunwald $E$, Jensen MD, Pories W, Fahrbach $K$, Schoelles K. Bariatric surgery: a systematic review and meta-analysis. JAMA. 2004;292(14):1724-37.

14. Groen VA, Graaf VA, Scholtes VA, Sprague S, Wagensveld BA, Poolman RW. Effects of bariatric surgery for knee complaints in (morbidly) obese adult patients: a systematic review. Obes Rev. 2015;16(2):161-70. https://doi.org/ 10.1111/obr.12236

15. Edwards C, Rogers A, Lynch S, Pylawka T, Silvis M, Chinchilli V, Mosher T, Black K. The effects of bariatric surgery weight loss on knee pain in patients with osteoarthritis of the knee. Arthritis. 2012;2012 https://doi.org/10.1155/ 2012/504189.

16. Richette P, Poitou C, Garnero P, Vicaut E, Bouillot JL, Lacorte JM, Basdevant A, Clément K, Bardin T, Chevalier X. Benefits of massive weight loss on symptoms, systemic inflammation and cartilage turnover in obese patients with knee osteoarthritis. Ann Rheum Dis. 2011;70(1):139-44. https://doi.org/ 10.1136/ard.2010.134015

17. Trofa D, Smith EL, Shah V, Shikora S. Total weight loss associated with increased physical activity after bariatric surgery may increase the need for total joint arthroplasty. Surg Obes Relat Dis. 2014;10(2):335-9. https://doi. org/10.1016/j.soard.2013.09.011.

18. Hidalgo JE, Roy M, Ramirez A, Szomstein S, Rosenthal RJ. Laparoscopic sleeve gastrectomy: a first step for rapid weight loss in morbidly obese patients requiring a second non-bariatric procedure. Obes Surg. 2012;22(4): 555-9. https://doi.org/10.1007/s11695-011-0574-z.

19. Severson EP, Singh JA, Browne JA, Trousdale RT, Sarr MG, Lewallen DG. Total knee arthroplasty in morbidly obese patients treated with bariatric surgery: a comparative study. J Arthroplast. 2012;27(9):1696-700. https://doi.org/10. 1016/j.arth.2012.03.005.

20. Roos EM, Roos HP, Lohmander LS, Ekdahl C, Beynnon BD. Knee injury and Osteoarthritis Outcome Score (KOOS) — development of a self-administered outcome measure. J Orthop Sports Phys Ther. 1998;28(2):88-96.

21. Roos EM, Toksvig-Larsen S. Knee injury and Osteoarthritis Outcome Score (KOOS) - validation and comparison to the WOMAC in total knee replacement. Health Qual Life Outcomes. 2003;1(1):17.

22. Roos EM, Lohmander LS. The Knee injury and Osteoarthritis Outcome Score (KOOS): from joint injury to osteoarthritis. Health Qual Life Outcomes. 2003; 1(1):64.

23. Aills L, Blankenship J, Buffington C, Furtado M, Parrott J. ASMBS allied health nutritional guidelines for the surgical weight loss patient. Surg Obes Relat Dis. 2008:4(5):S73-108. https://doi.org/10.1016/.jsoard.2008.03.002.

24. Katz S, Ford AB, Moskowitz RW, Jackson BA, Jaffe MW. Studies of illness in the aged. The index of ADL: a standardized measure of biological and psychological function. JAMA. 1963;185:914-9.

25. Almangoush A, Herrington L, Attia I, Jones R, Aldawoudy A, Aziz AA, Waley A. Cross-cultural adaptation, reliability, internal consistency and validation of the Arabic version of the Knee injury and Osteoarthritis Outcome Score (KOOS) for Egyptian people with knee injuries. Osteoarthr Cartil. 2013;21(12): 1855-64. https://doi.org/10.1016/j.joca.2013.09.010.

26. Orthopaedic Score, http://www.orthopaedicscore.com. Accessed 16 May 2016.

27. Stata, Data Analysis and Statistical Software, http://www.stata.com. Accessed 16 May 2016.

28. Thomeé R, Augustsson J, Karlsson J. Patellofemoral pain syndrome: a review of current issues. Sports Med. 1999;28(4):245-62.

29. Roush JR, Bay RC. Prevalence of anterior knee pain in 18-35 year-old females. Int J Sports Phys Ther. 2012;7(4):396.

30. Zalesin KC, Franklin BA, Lillystone MA, Shamoun T, Krause KR, Chengelis DL, Mucci SJ, Shaheen KW, McCullough PA. Differential loss of fat and lean mass in the morbidly obese after bariatric surgery. Metab Syndr Relat Disord. 2010;8(1):15-20. https://doi.org/10.1089/met.2009.0012.

31. Stegen S, Derave W, Calders P, Van Laethem C, Pattyn P. Physical fitness in morbidly obese patients: effect of gastric bypass surgery and exercise training. Obes Surg. 2011;21(1):61-70. https://doi.org/10.1007/s11695-009-0045-y.

\section{Ready to submit your research? Choose BMC and benefit from:}

- fast, convenient online submission

- thorough peer review by experienced researchers in your field

- rapid publication on acceptance

- support for research data, including large and complex data types

- gold Open Access which fosters wider collaboration and increased citations

- maximum visibility for your research: over $100 \mathrm{M}$ website views per year

At BMC, research is always in progress.

Learn more biomedcentral.com/submissions 